\title{
Application of Fourier transform infrared (FT-IR) spectroscopy combined with chemometrics for authentication of cod-liver oil.
}

\begin{abstract}
Some vegetable oils such as canola $(\mathrm{CaO})$, corn $(\mathrm{CO})$, soybean $(\mathrm{SO})$, and walnut (WO) oils have similar color with cod liver oil (CLO), therefore, the presence of these oils was difficult to detect using naked eye. For this reason, Fourier transform infrared (FTIR) spectroscopy using horizontal attenuated total reflectance (HATR) as sampling accessory and in the combination with chemometrics was developed for detection and quantification of these vegetable oils as adulterants in CLO. The quantification of vegetable oils was carried out by using multivariate calibrations of partial least squares (PLS) and principle component regression (PCR), while the classification between pure CLO and CLOs adulterated with $\mathrm{CaO}, \mathrm{CO}$, SO, and WO was performed using discriminant analysis (DA). PLS with FTIR normal spectra was more suitable compared with PCR for quantification purposes with coefficient of determination (R2) higher than 0.99 and root mean square error of calibration (RMSEC) in the range of $0.04-0.82 \%(\mathrm{v} / \mathrm{v})$. The PLS model was further used to predict the levels of these vegetable oils in independent samples for validation/prediction purpose. The root mean square error of prediction (RMSEP) values obtained were of $1.75 \%(\mathrm{v} / \mathrm{v})(\mathrm{CaO})$, $1.39 \%(\mathrm{v} / \mathrm{v})(\mathrm{CO}), 1.35 \%(\mathrm{v} / \mathrm{v})(\mathrm{SO})$, and $1.37 \%(\mathrm{v} / \mathrm{v})(\mathrm{WO})$, respectively. The classification using DA revealed that the developed method can classify CLO and that mixed with these vegetable oils using 9 principal components.
\end{abstract}

Keyword: Authentication; Cod liver oil; FTIR spectroscopy; Multivariate calibration; Discriminant analysis. 